\title{
Effects of Higher Order Dispersion Terms in the Nonlinear Schrodinger Equation
}

\author{
Robert Beech and Frederick Osman \\ University of Western Sydney, Penrith-South, 1791, Australia
}

\begin{abstract}
This study presents a concise graphical analysis of solitonic solutions to a nonlinear Schrodinger equation (NLSE). A sequence of code using the standard NDSolve function has been developed in Mathematica to investigate the acceptable accuracy of the NLSE in relatively small ranges of the dispersive parameter space. An operator splitting approach was used in the numerical solutions to expand the boundaries and reduce the artifacts for a reliable solution. These numerical routines were implemented through the use with Mathematica and the results give a very clear view of this interesting and important practical phenomenon.
\end{abstract}

Key words: Solitons, Solitonic solutions, Nonlinear Schrodinger equation, Numerical artifacts

\section{INTRODUCTION}

The nonlinear Schrödinger equation (NLSE):

$\mathrm{i} \frac{\partial \mathrm{u}}{\partial \xi}+\frac{1}{2} \frac{\partial^{2} \mathrm{u}}{\partial \tau^{2}}+\mathrm{Abs}\left[\mathrm{u}^{2}\right] \mathrm{u}=-\mathrm{i} \Gamma \mathrm{u}+\mathrm{i} \alpha \frac{\partial^{3} \mathrm{u}}{\partial \tau^{3}}$

May be used to model the propagation of pulsed light in optical fibres ${ }^{[1,2]}$. When $\Gamma=\alpha=0$ this becomes the standard model of picosecond pulses ${ }^{[3]} . \mathrm{u}(\xi, \tau)$ is a complex-valued function modelling the envelope of the effective electric field as a function of distance $\xi$ along the fiber and time $\tau$ in a frame moving at the group velocity. The left-hand side of Equation (1) models the effects of group velocity dispersion and self-phase modulation while the right-hand side accounts for the refractive nature of the fiber (with refractive index $\Gamma$ ) and higher order linear dispersion effects (with coefficient $\alpha$ ). As an initial attempt to understand the contribution of the dispersion term, results for non-zero $\alpha$ (and $\Gamma=0$ ) will be compared to the well-known soliton solution of the standard model. Defining the NLSE operator as:

$$
\begin{aligned}
& \operatorname{nlse}\left[\alpha_{-}, n_{-}\right]\left[u_{-}\right]:=i \partial_{\xi} u+1 / 2 \partial_{(\tau, 2)} u+A b s[u]^{2} u \\
& -i \alpha \partial_{(\tau, n)} u
\end{aligned}
$$

the fundamental Zakharov-Shabat soliton solution of the standard model ${ }^{[4]}$ can be verified:

$$
\mathrm{u}(\xi, \tau)=\operatorname{Sech}(\tau) \mathrm{e}^{\mathrm{i} \xi / 2}
$$

Simplify[nlse[0,0][Sech[ $\tau] \operatorname{Exp}[i \xi / 2]]==0,\{\xi, \tau\} \in$

Reals]: The standard methods available with Mathematica's NDSolve function did not provide sufficient accuracy for our analysis. Rob Knapp has kindly provided a package

$\mathrm{u}[0, \tau]=\operatorname{Sech}[\tau]$

based on a split step method used in this study. The NLSE with $n^{\text {th }}$ order dispersion is solved numerically with initial condition and asymmetric boundary condition:

$\mathrm{u}(\xi,-\tau \lim )=\mathrm{u}(\xi, \tau \lim )$

which models a train of interacting pulses.

Function definitions: The standard NDSolve method of StiffnessSwitching is implemented in the following function:

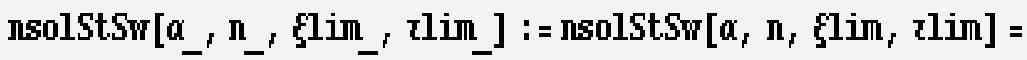

$$
\begin{aligned}
& \text { IIDSolve }[\{\mathrm{nlse}[\alpha, \mathrm{n}][u[\xi, \tau]]=\mathbf{0}, \mathbf{u}[0, \tau]==\operatorname{Sech}[\tau], \mathbf{u}[\xi,-\tau \lim ]==\mathbf{u}[\xi, \tau \lim ]\} \text {, } \\
& u[\xi, \tau],\{\tau,-\tau \lim , \tau \lim \},\{\xi, 0, \xi l i m\}, \text { Method } \rightarrow \text { StiffnessSwitching, } \\
& \text { MaxSteps } \rightarrow \text { Infinity, PrecisionGoal } \rightarrow \text { 2][[1]] }
\end{aligned}
$$

The non-standard Split Step method is implemented in the following function: 


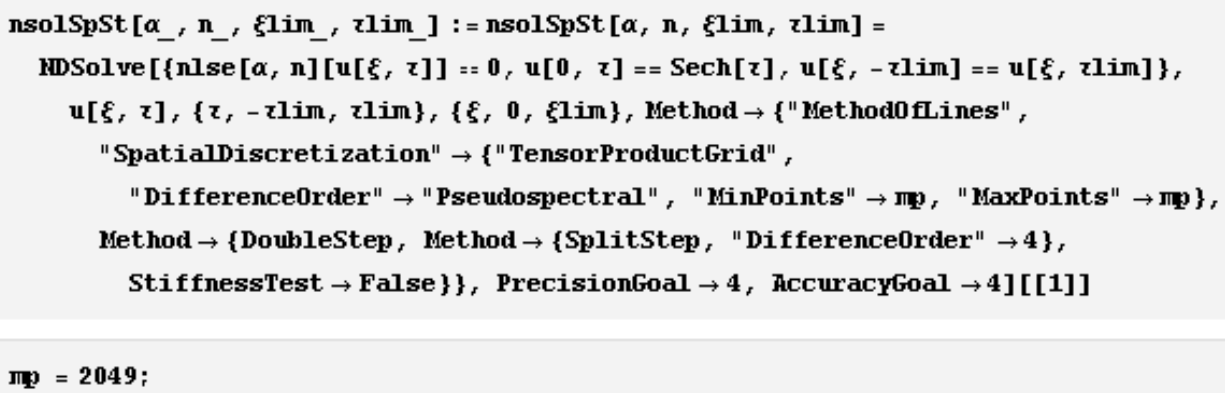

The following functions produce amplitude, contour and $\tau$ and $\xi$ cross-section plots for the NLSE solution:

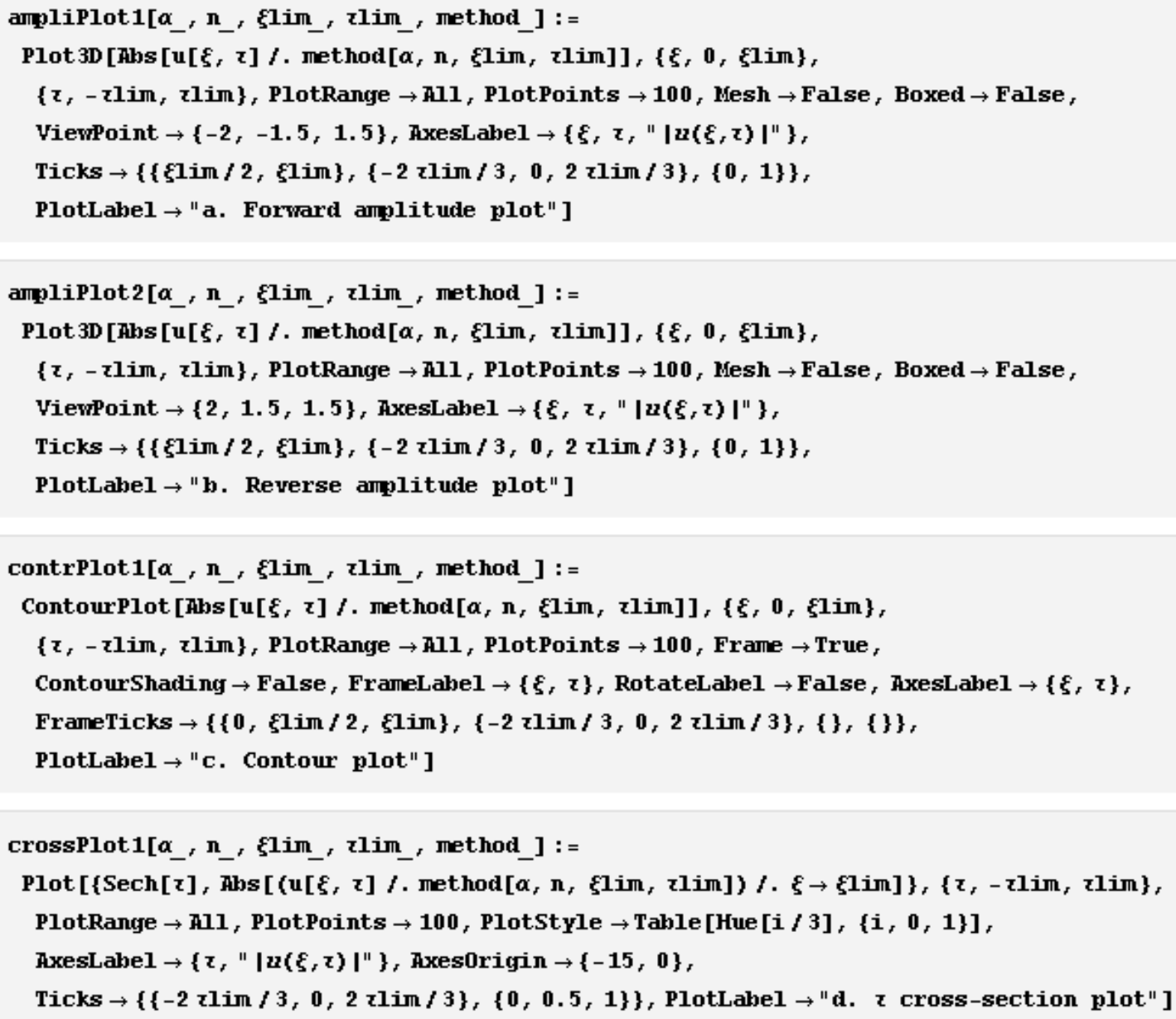

This function displays the plots: 


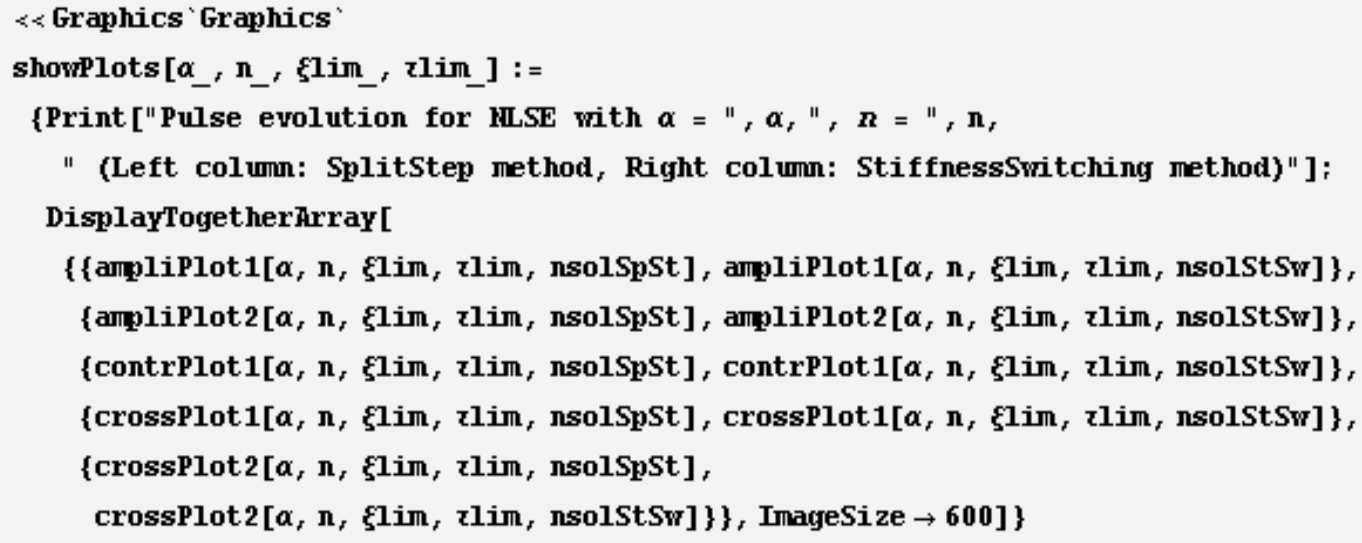

These functions produce a series of $\tau$ cross-section plots (using the Split Step method) for animation:

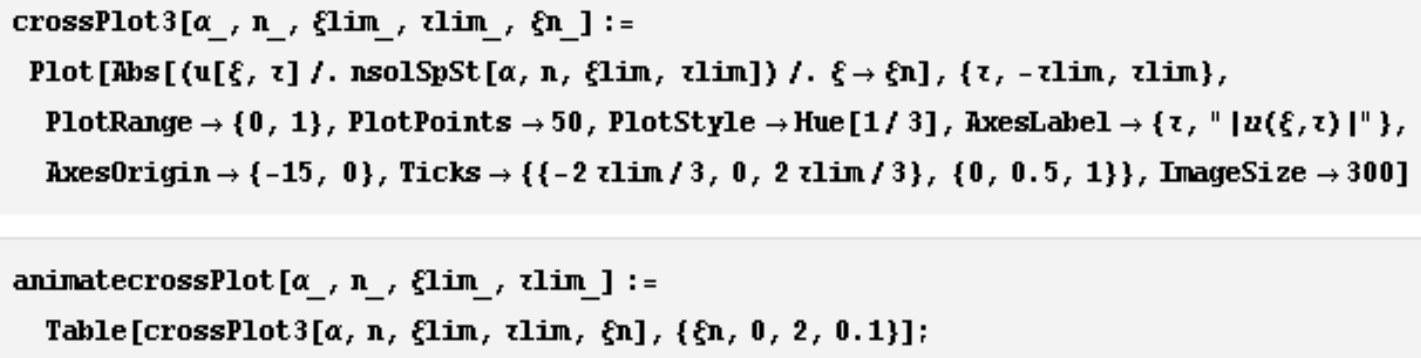

Results of Split Step and StiffnessSwitching cases plotted side by side: The following plots explore the effect of order $n$ linear dispersion. The dispersion coefficient $\alpha$ is chosen to produce representative examples behavior e observed behavior. On the left side of the graphic columns are the Split Step method graphs. The plots on the right show the results for the StiffnessSwitching method. In each case, plots a. and b. Illustrate the evolution of the soliton for $0 \leq \xi \leq \xi \lim$, plot (a) showing the forward amplitude and plot (b) the reverse. Plot (c) shows the associated contour plot. Plot (d) shows two crosssectional profiles along the $\tau$ axis, the direction of motion of the wave, and compares the initial pulse at $\xi=0$ (red) with the pulse obtained at $\xi=\xi \lim$ (green). Finally, plot (e) gives the corresponding cross-section along the $\xi$ axis and indicates the deviation along the crest of the wave of the final pulse compared to the initial pulse.

For the Split Step method PrecisionGoal was set at 4 to achieve suitable accuracy for $n>0$. The maximum achievable consistent value of PrecisionGoal was 2 for the StiffnessSwitching method.

\section{* No dispersion}

Zero order: Soliton solution remains unchanged. No apparent difference between methods.

\section{* Higher order dispersion}

First order: Soliton solution is retarded. StiffnessSwitching method apparently introduces numerical artifacts.

Second order: Soliton solution disperses symmetrically.

Third order, low $\alpha$ : Nonsymmetric radiation produced, possible numerical artifacts appearing as transverse waves originating on $\tau$ boundaries.

Third order, higher $\alpha$ : Nonsymmetric radiation increased and feeding into a pulse.

Fourth order: Symmetric dispersion, possible small radiation or numerical artifacts.

Fifth order: Increasing $\alpha$ from 0.005 to 0.006 initiates nonconvergence of StiffnessSwitching method before non-symmetric radiation becomes pronounced.

Sixth order: Increasing $\alpha$ past 0.009 initiates nonconvergence of numerical method, numerical anomaly evident in the plot. 
Pulse evolution for RLSE with $\alpha=0, n=0$

(Left column: $S_{p} l$ it Step method, Right column: Stiffnesswitching method)

2. Forward emplitude plot

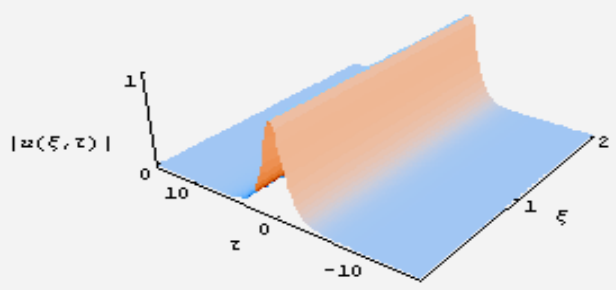

b. Reverse emplitude plot

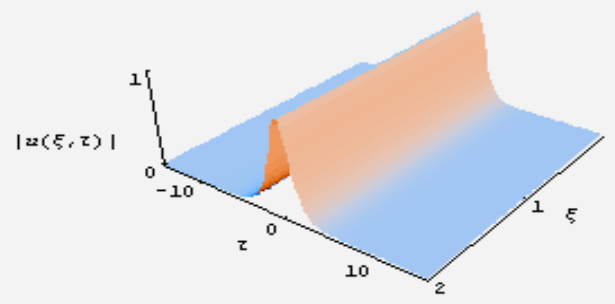

c. Contour plot
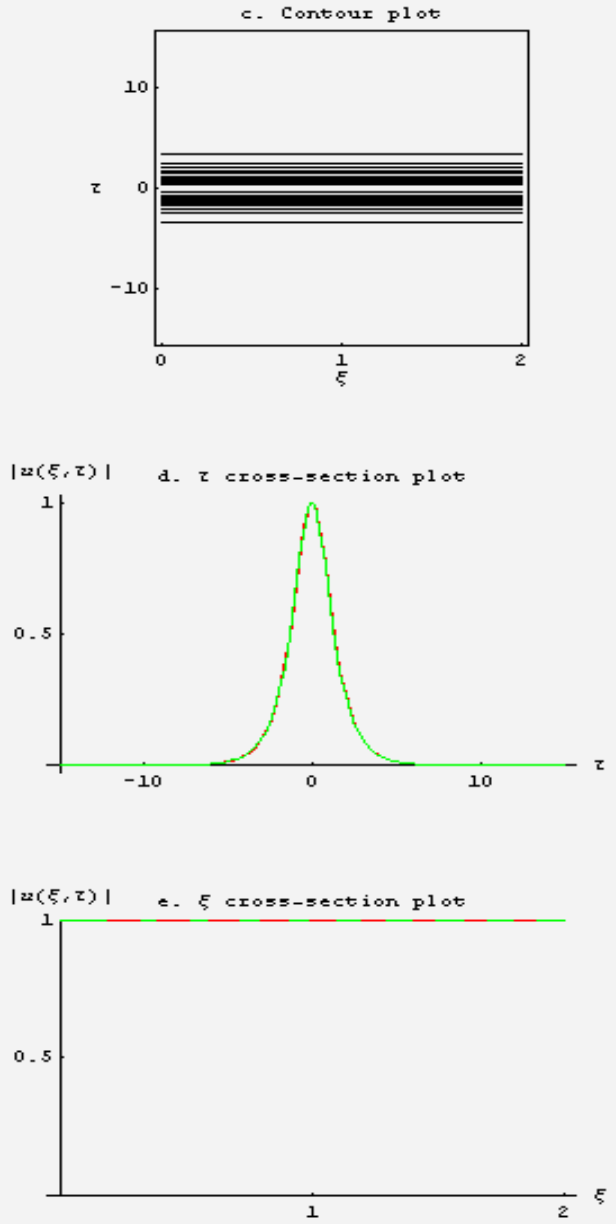

2. Forward emplitude plot

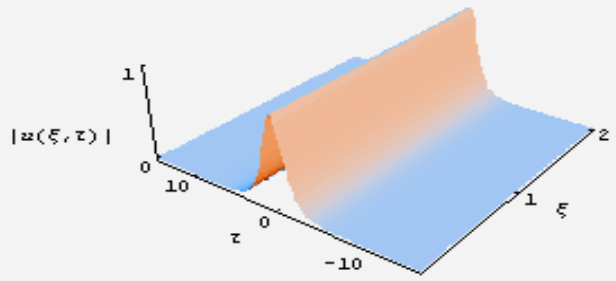

b. Reverse emplitude plot

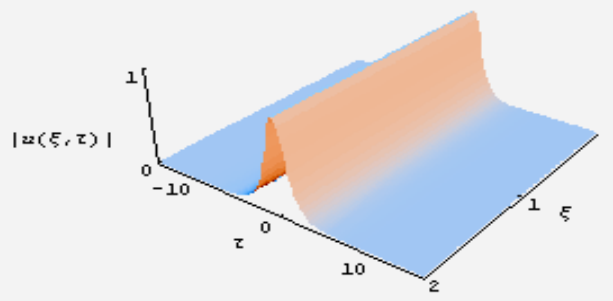

c. Contour plot
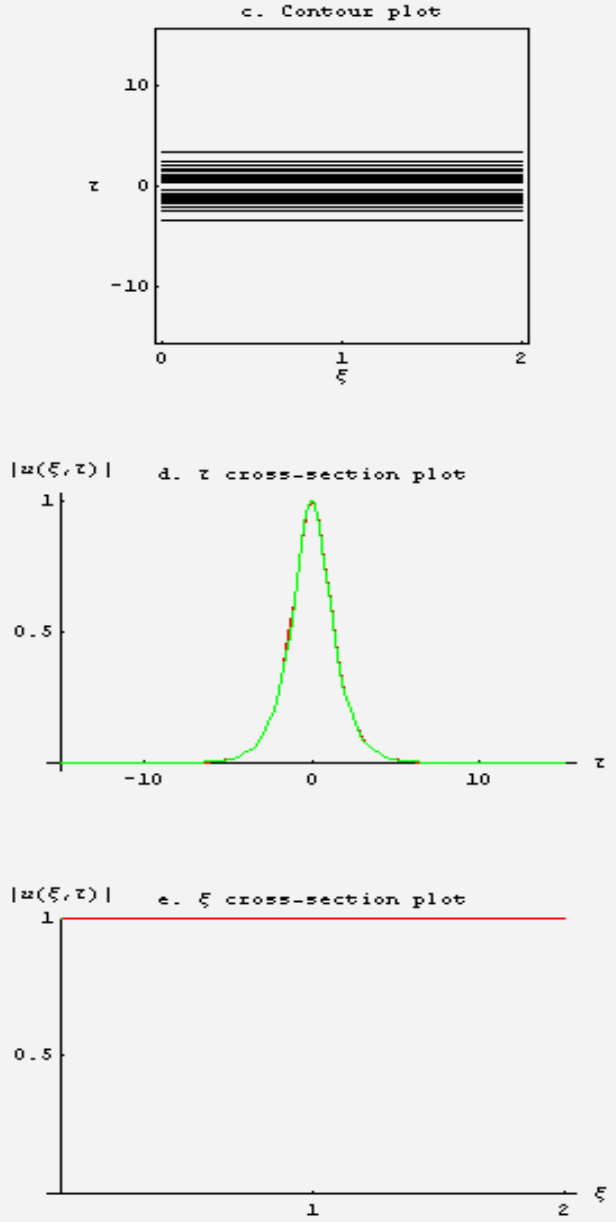
Pulse evolution for MLSE with $\alpha=1, \pi=1$

(Left column: $S_{P} 1$ it $S t e p$ method, Right column: Stiffnesswitching method)

2. Formard amplitude plot

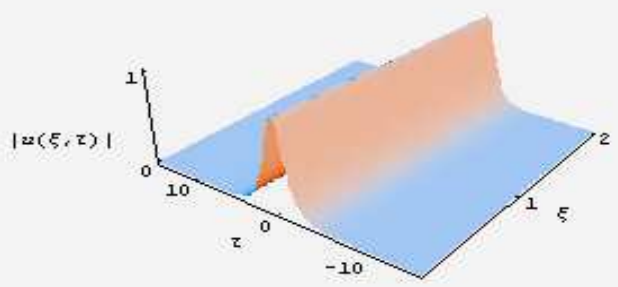

b. Reverse amplitude plot

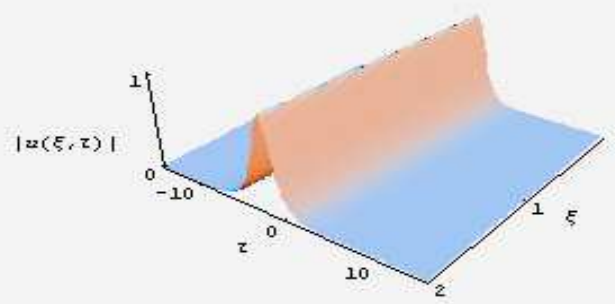

c. Contour $\mathrm{P}^{1}$ ot
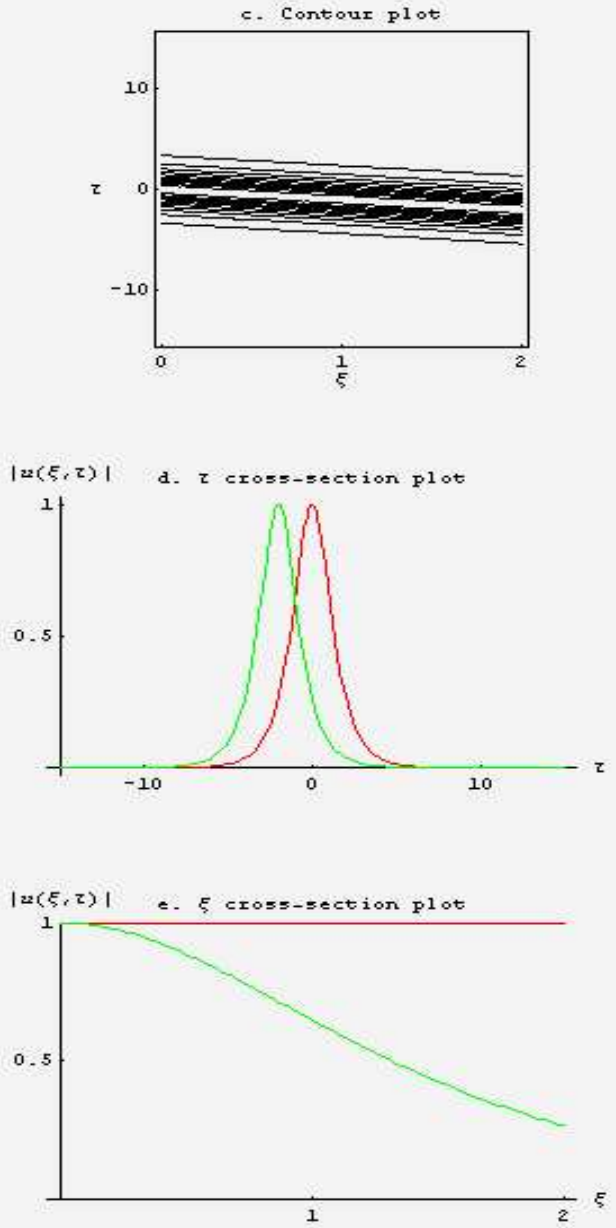

2. Forward emplitude plot

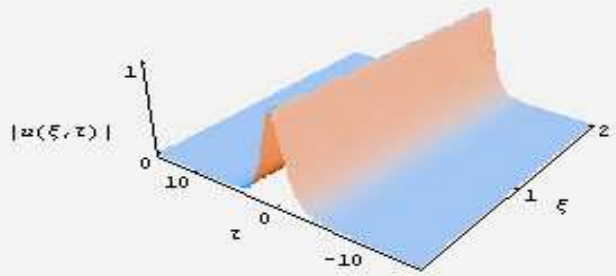

b. Reverse emplitude plot

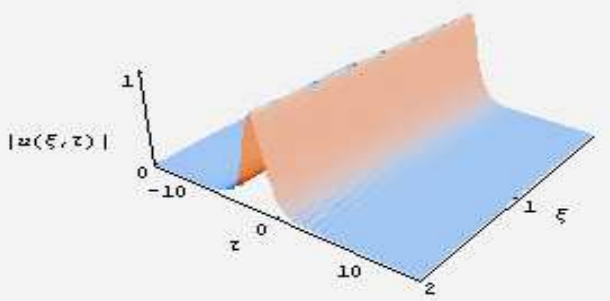

c. Contour plot
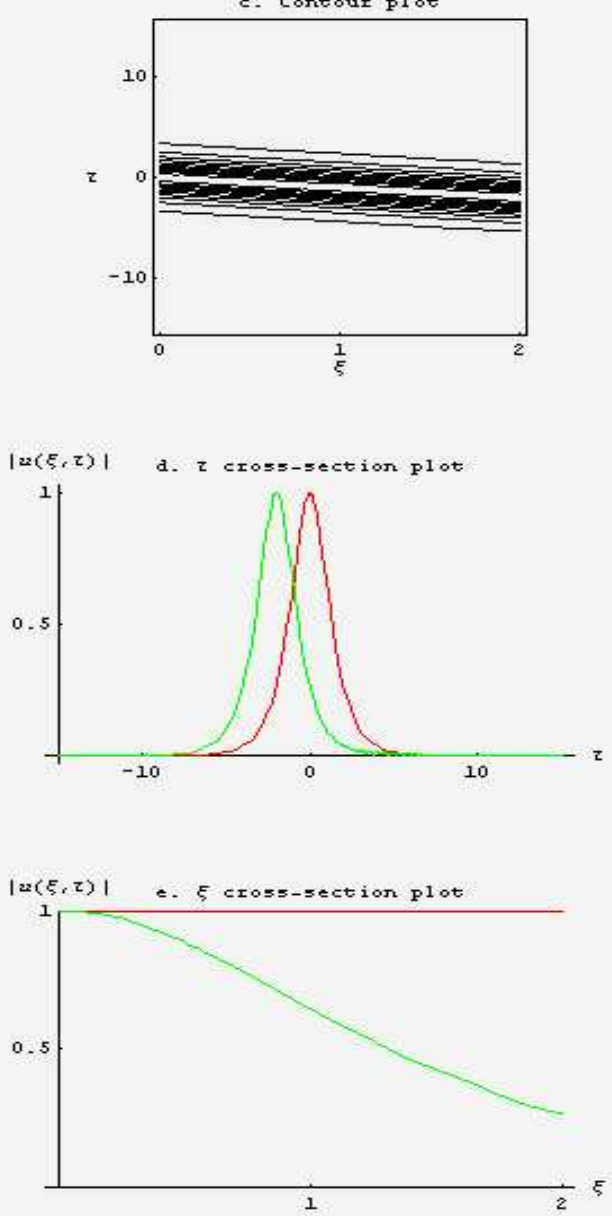
Pulse evolution for MLSE with $\alpha=1.8, z=1$

(Left column: SPlitSteP method, Right column: Stiffessswitching method)

2. Formard amplitude plot

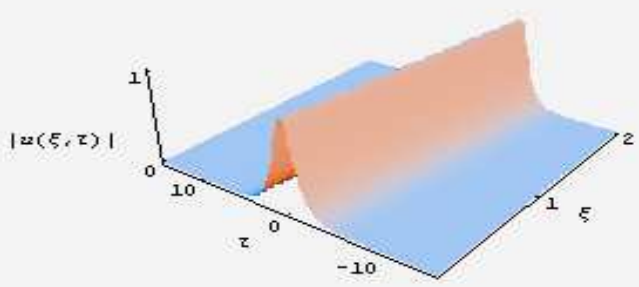

b. Reverse amplitude plot

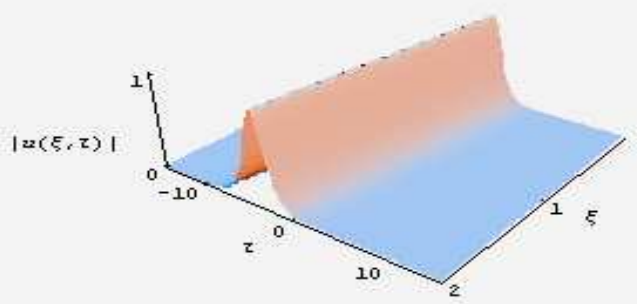

c. Contour plot
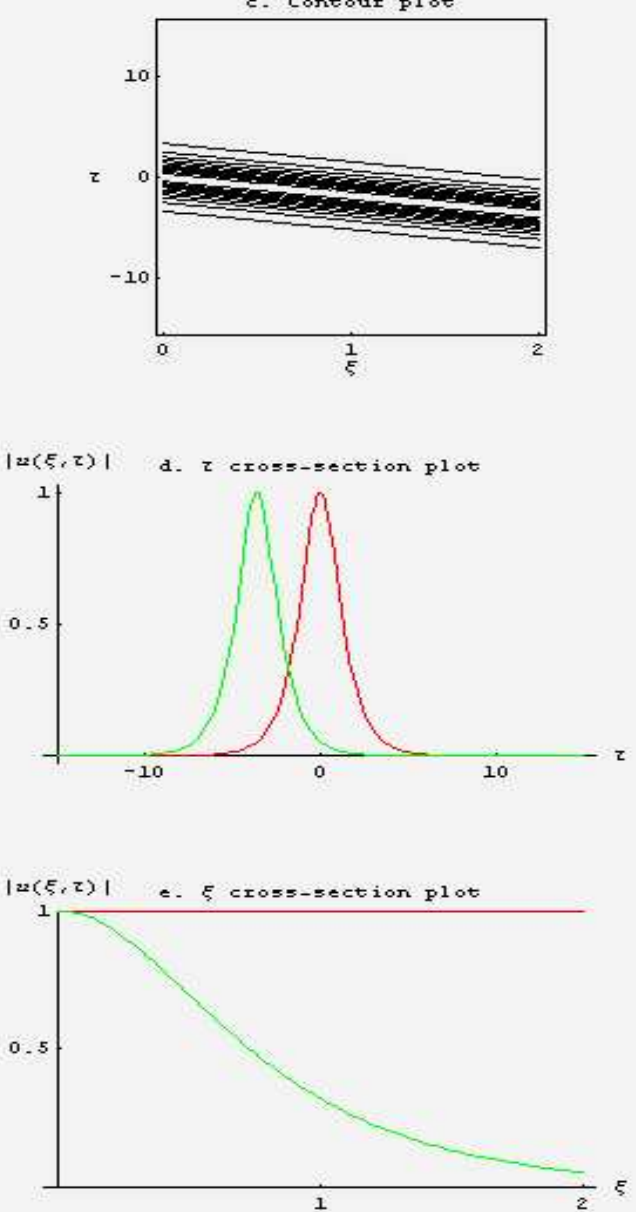

2. Forward amplitude plot

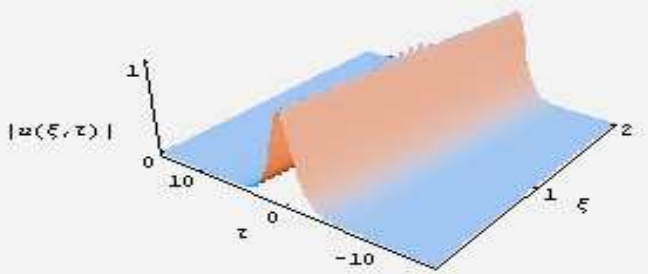

b. Reverse amplitude plot

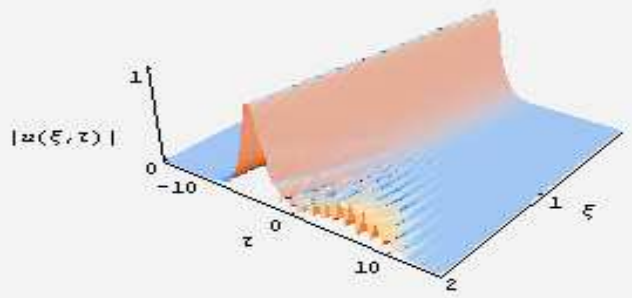

c. Contour plot
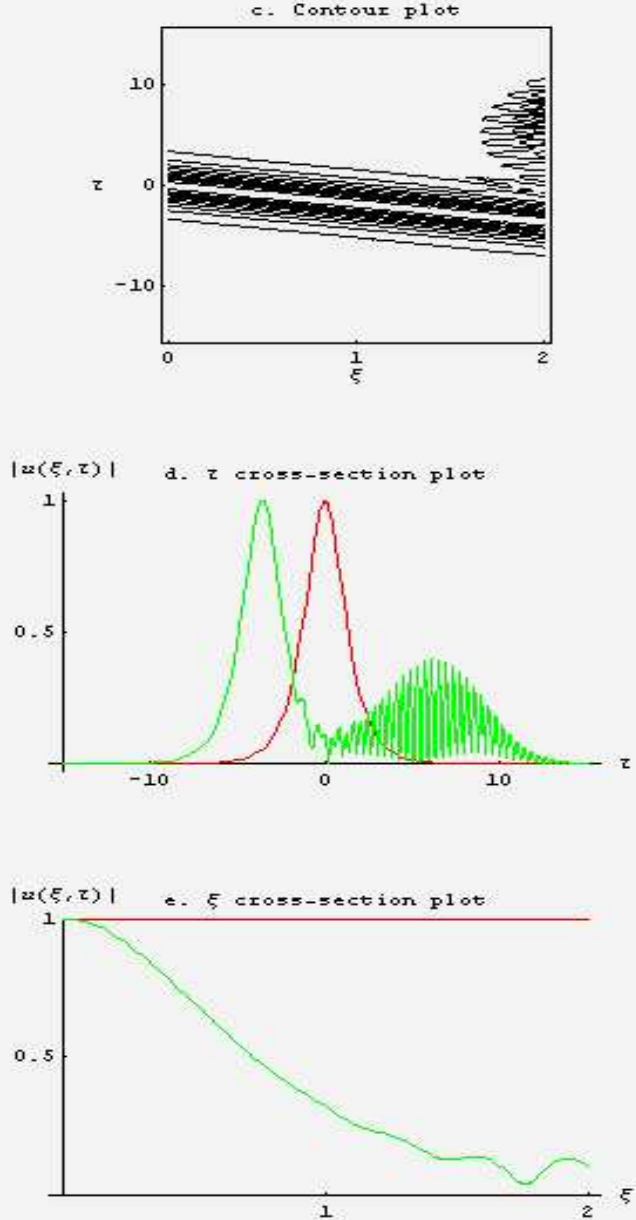
Pulse evolution for MLSE with $\alpha=1.5, n=2$

(Left column: Splitstep method, Right column: Stiffnesswitching method)

2. Forward amplitude plot

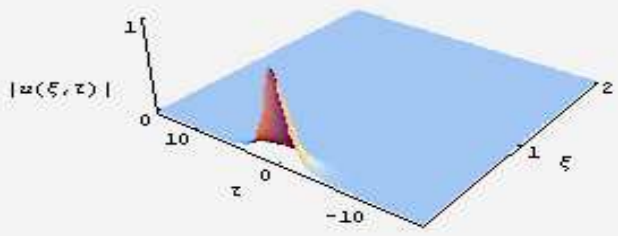

b. Reverse amplitude plot
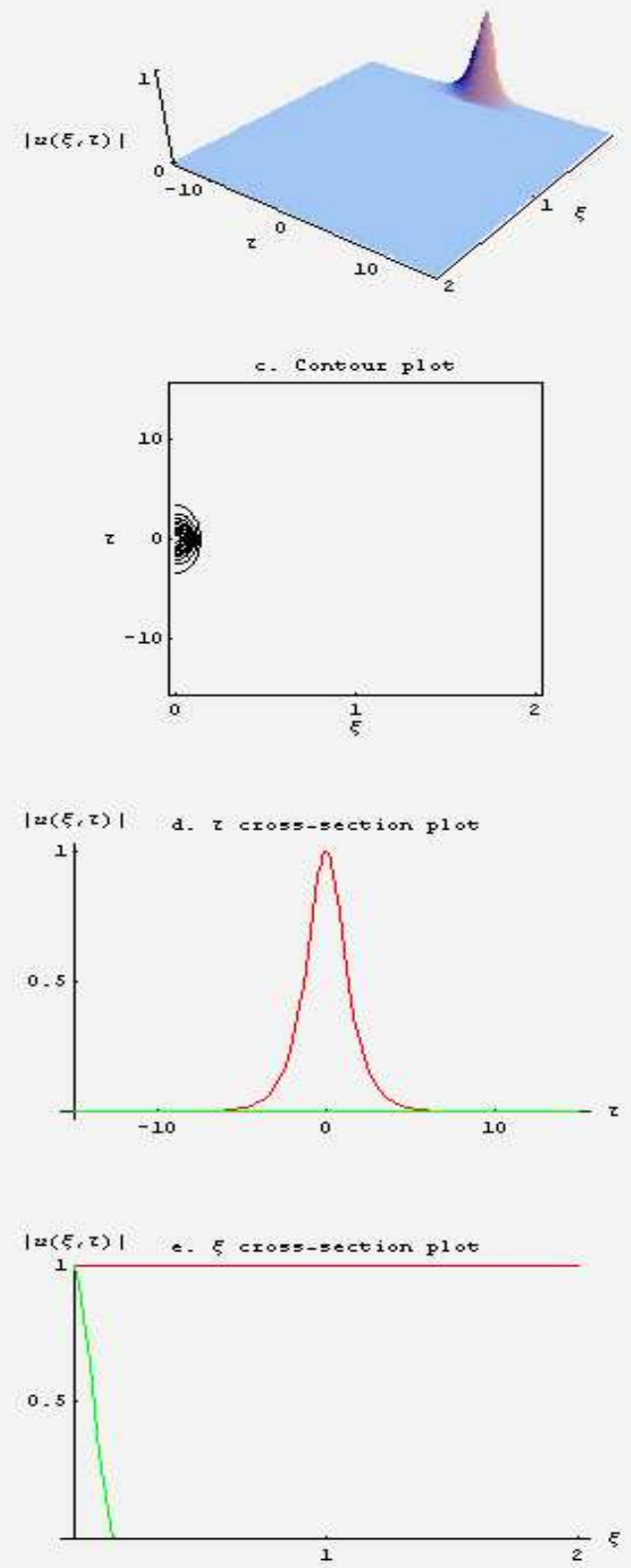

2. Forward amplitude plot

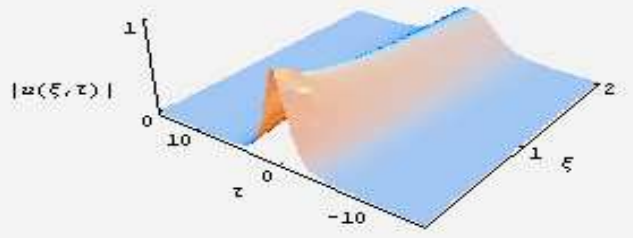

b. Reverse emplitude plot

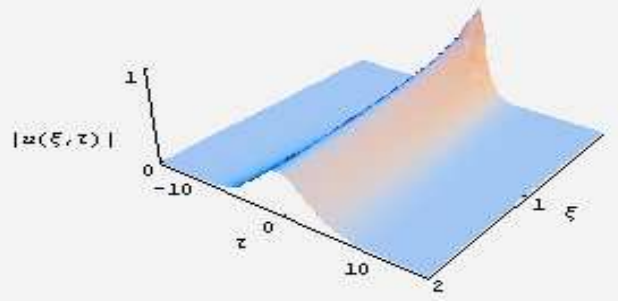

c. Contour plot
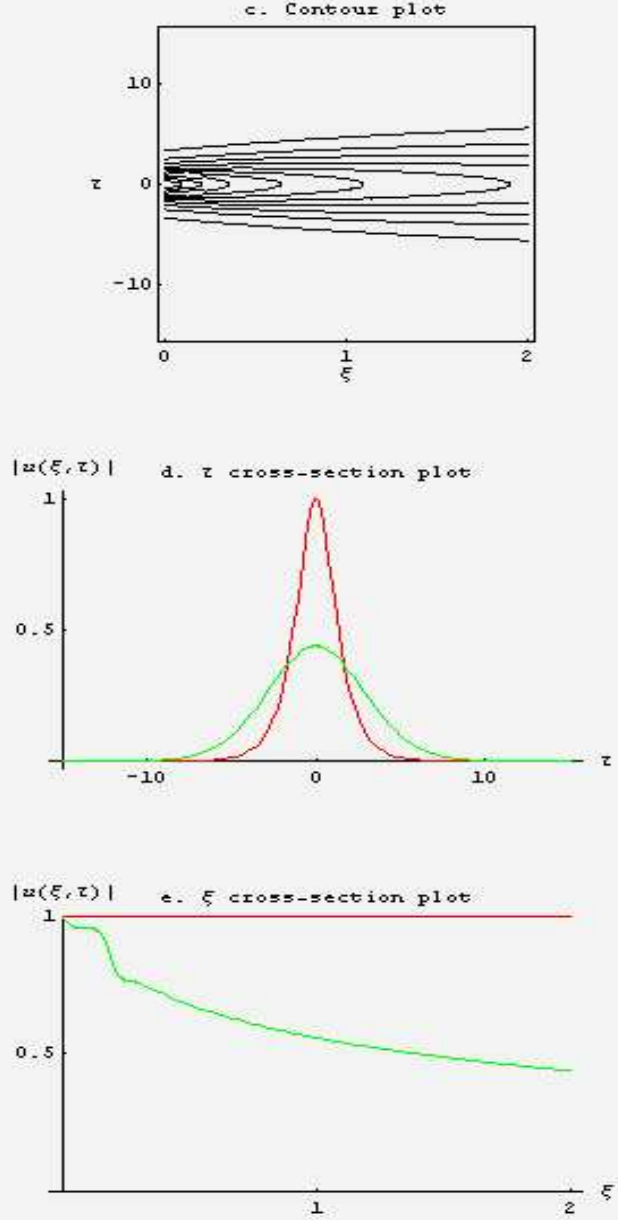
Pulse evolution for MLSE with $\alpha=0.05, n=3$

(Left column: Splitstep method, Right column: Stiffresswitching method)
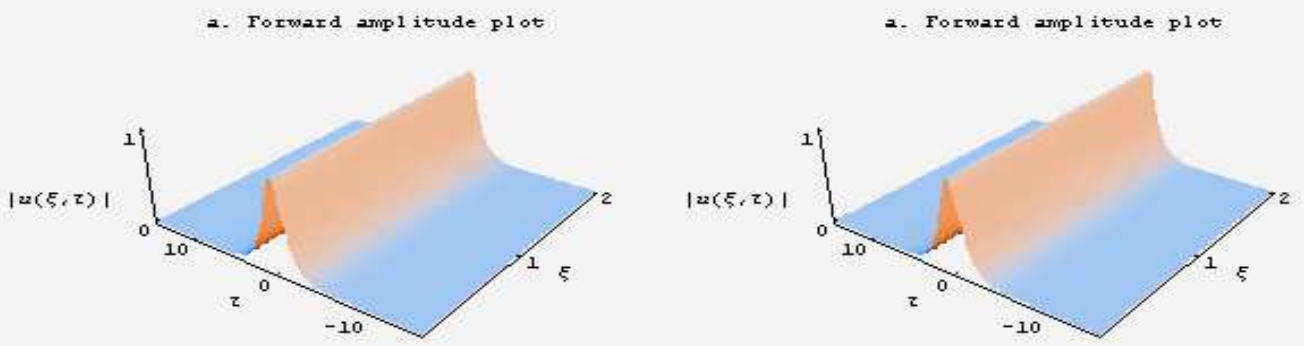

b. Reverse amplitude plot

b. Reverse 2mplitude plot
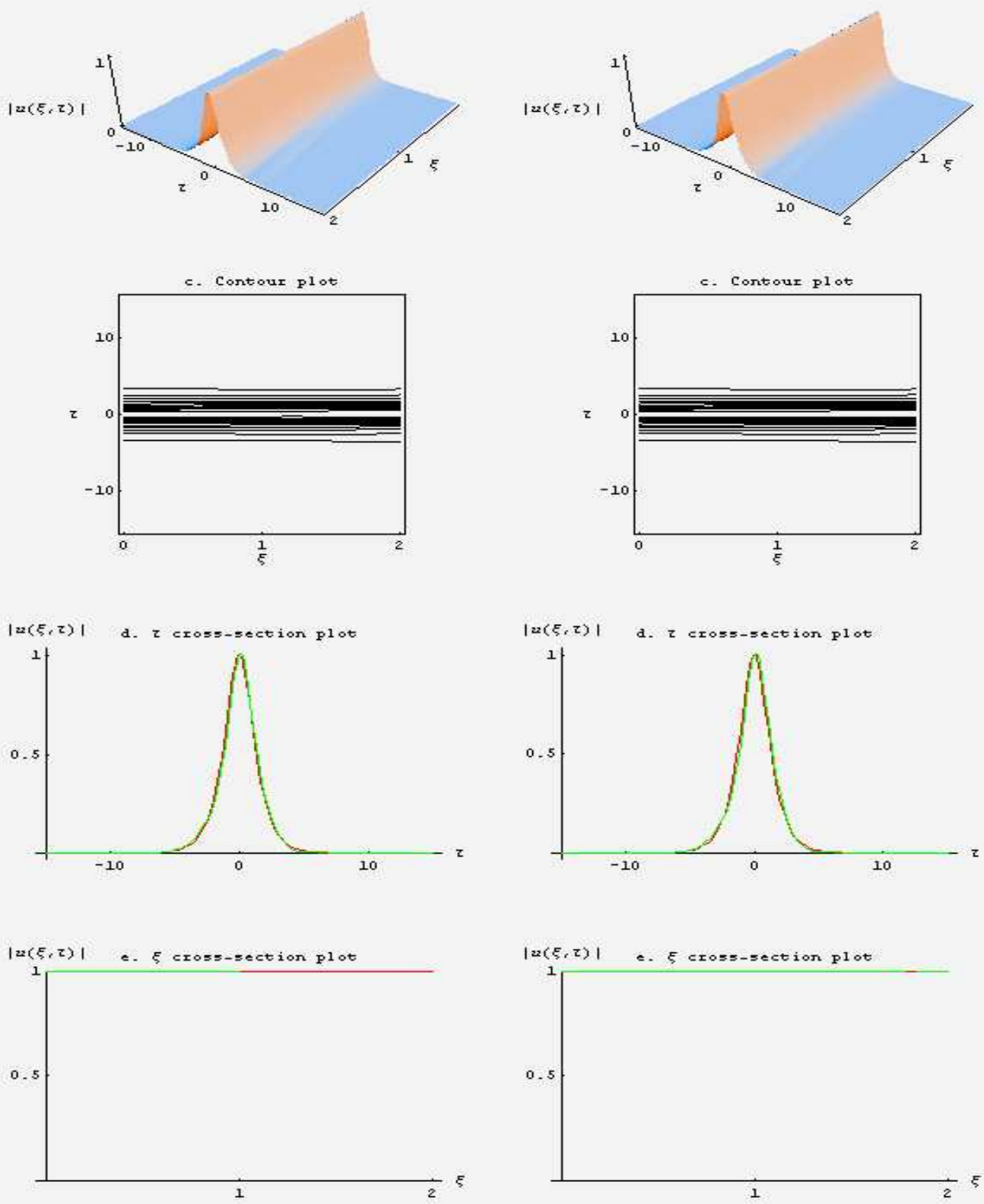
Pulse evolution for MLSE with $\alpha=1.5, n=3$

(Left column: Splitstep method, Right column: Stiffnesswitching method)

2. Forward amplitude plot

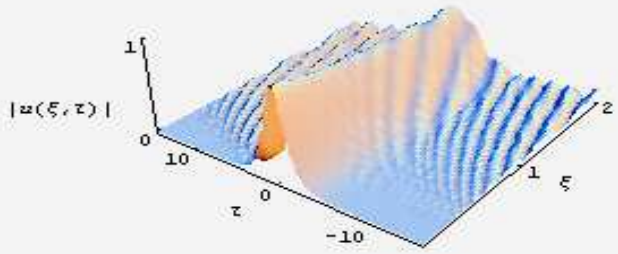

b. Reverse amplitude plot
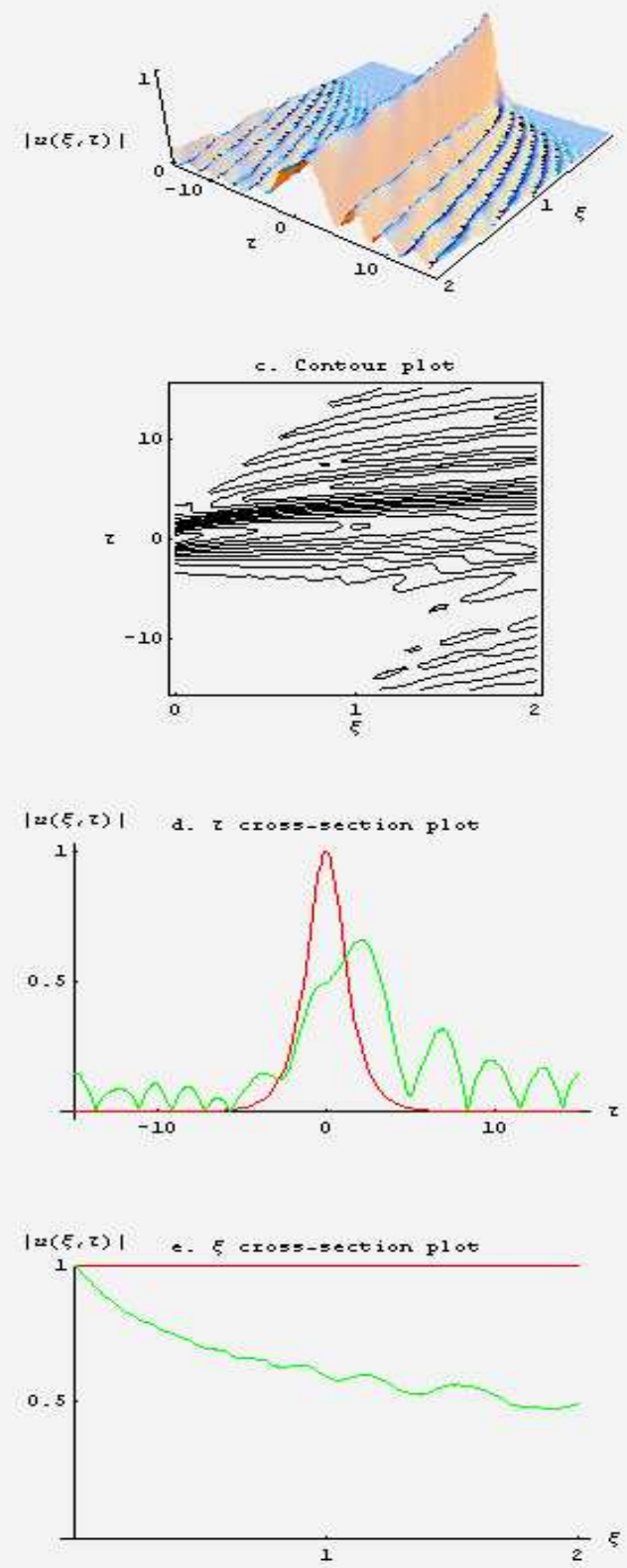

2. Forward amplitude plot

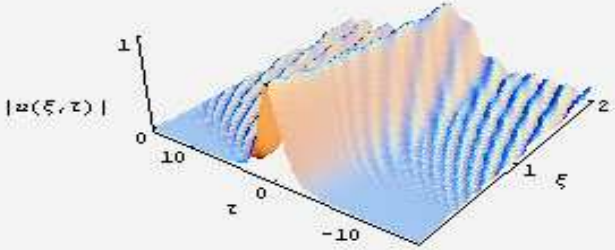

b. Reverse amplitude plot
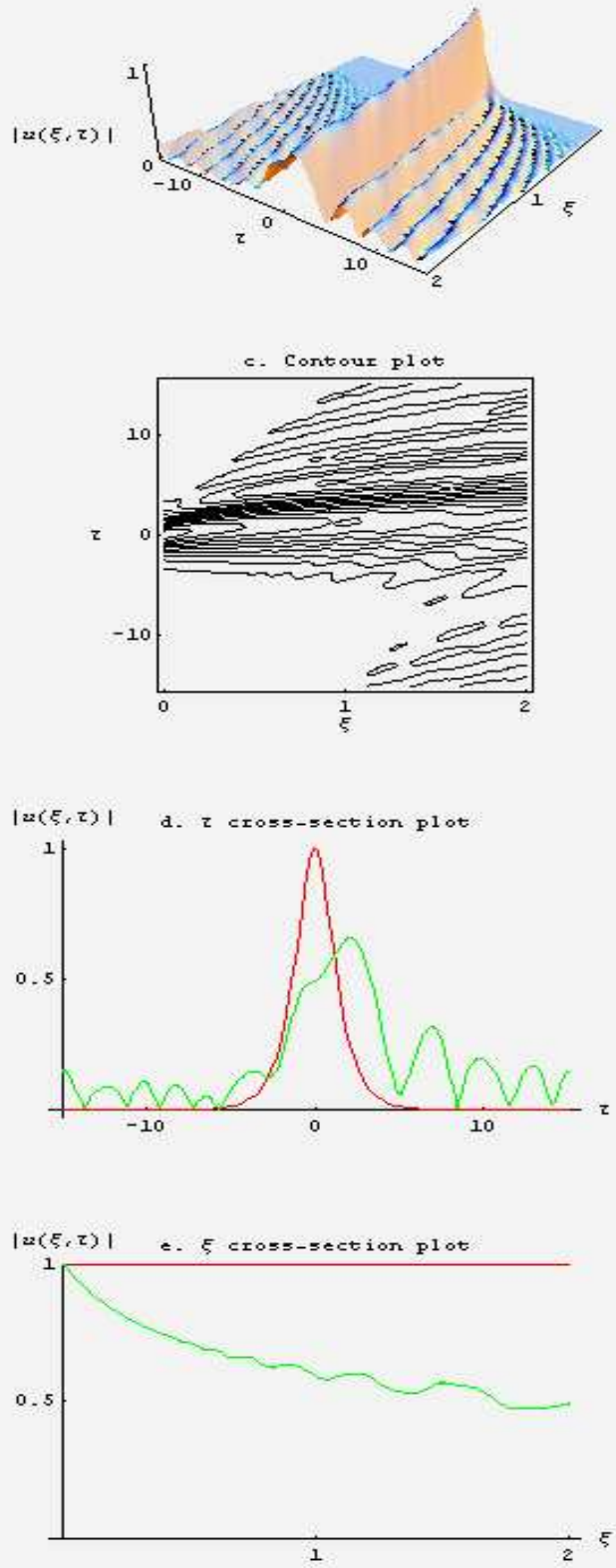
Pulse evolution for MLSE with $\alpha=0.007, \pi=4$

(Left column: SplitStep method, Right columo: Stiffnesswitching method)

2. Formard emplitude plot

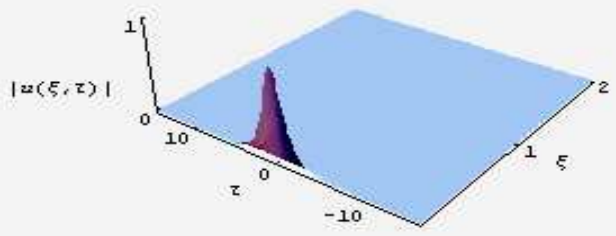

b. Reverse amplitude plot

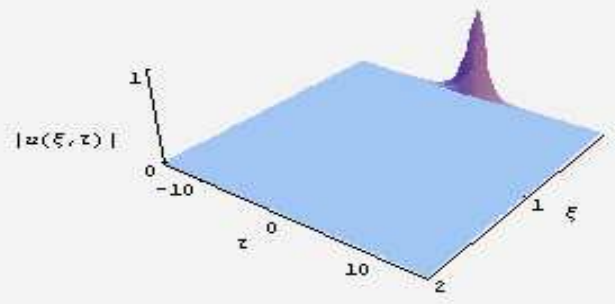

Contour plot
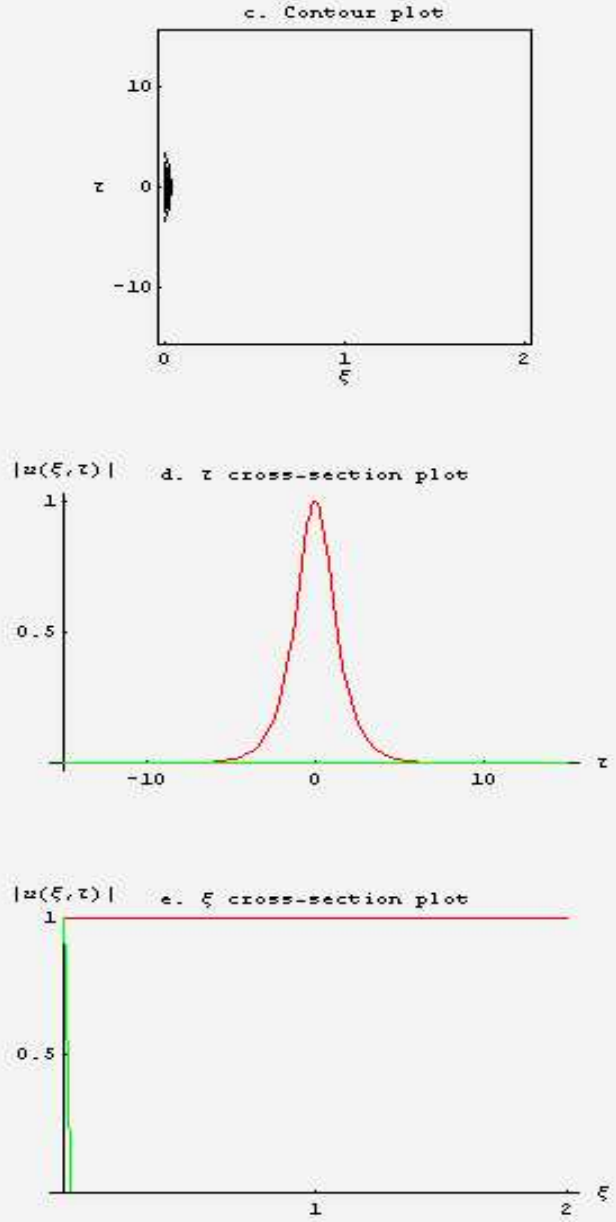

2. Forward amplitude plot

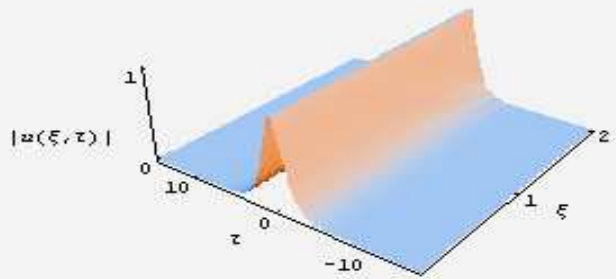

b. Reverse 2mplitude plot

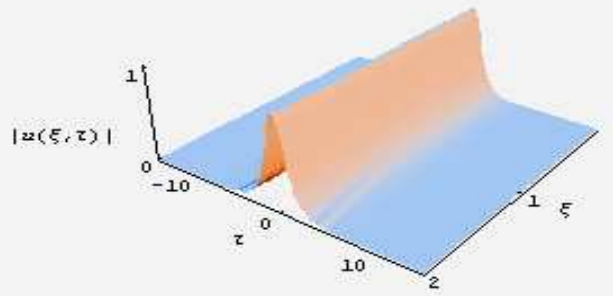

c. Contoux plot
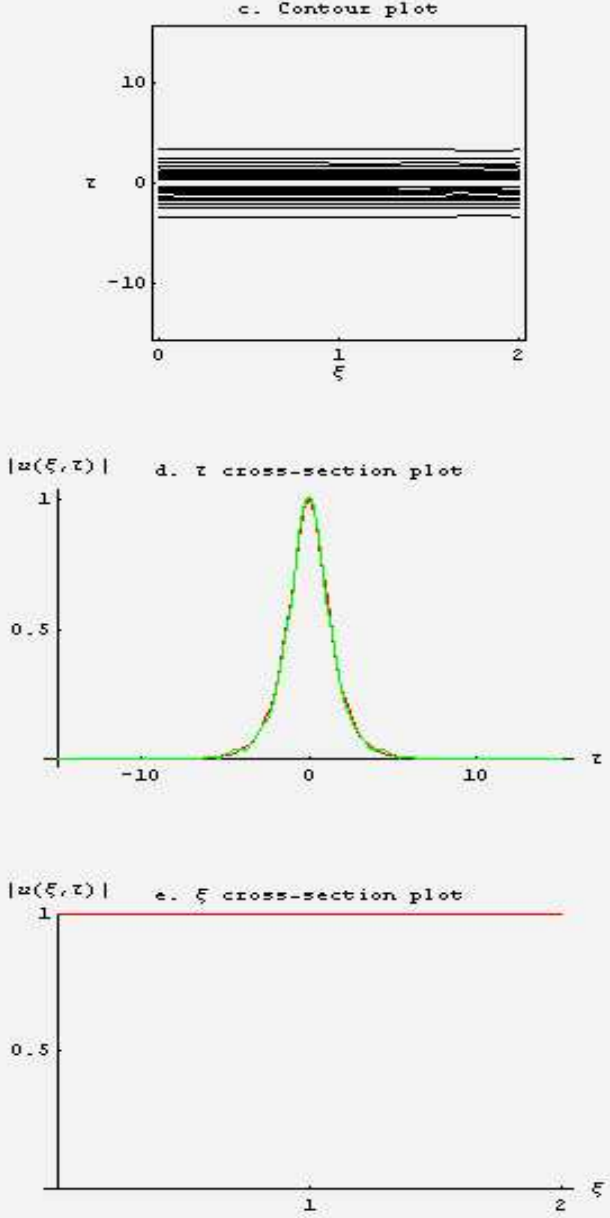
Pulse evolution for RLSE with $\alpha=0.005, \pi=5$

(Left column: SplitStep method, Right colume: Stiffessswitching method)

2. Formard amplitude plot

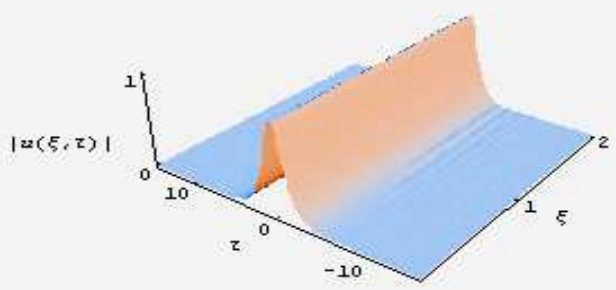

b. Reverse emplitude plot

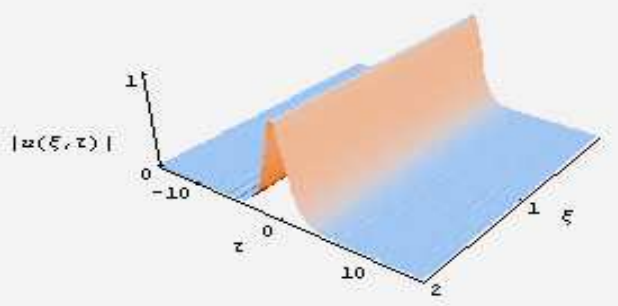

c. Contour plot
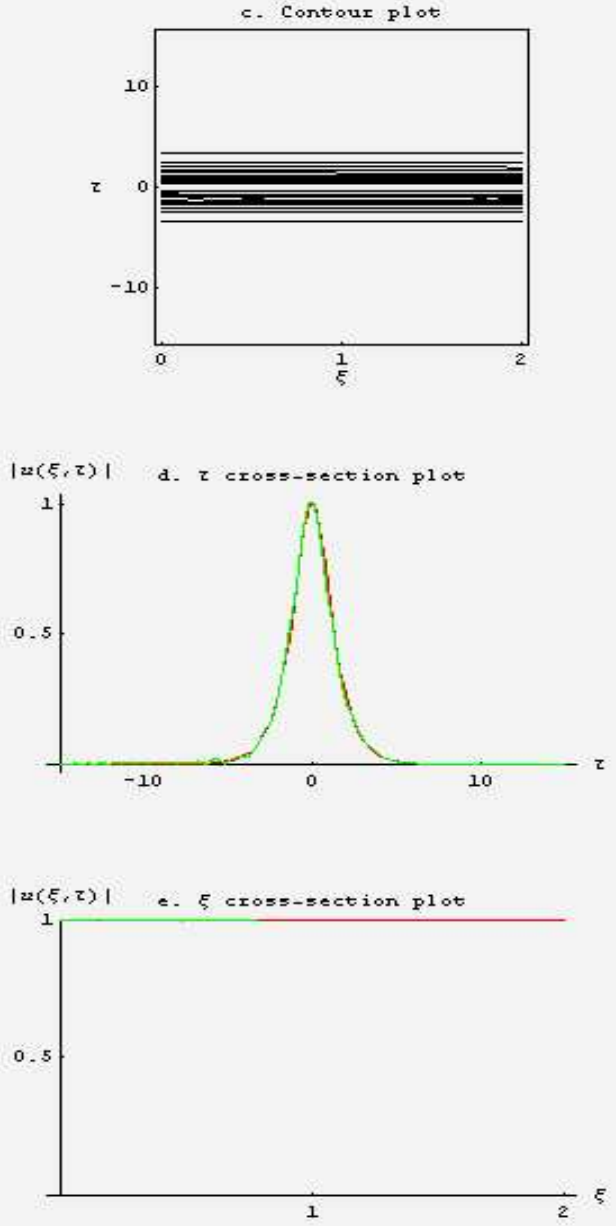

2. Formard amplitude plot

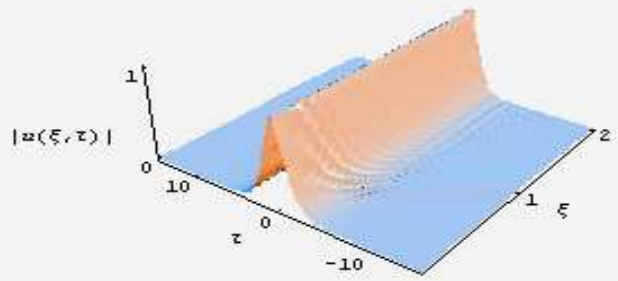

b. Reverse emplitude plot

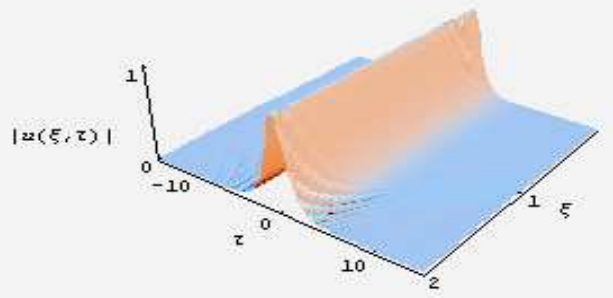

c. Contour plot
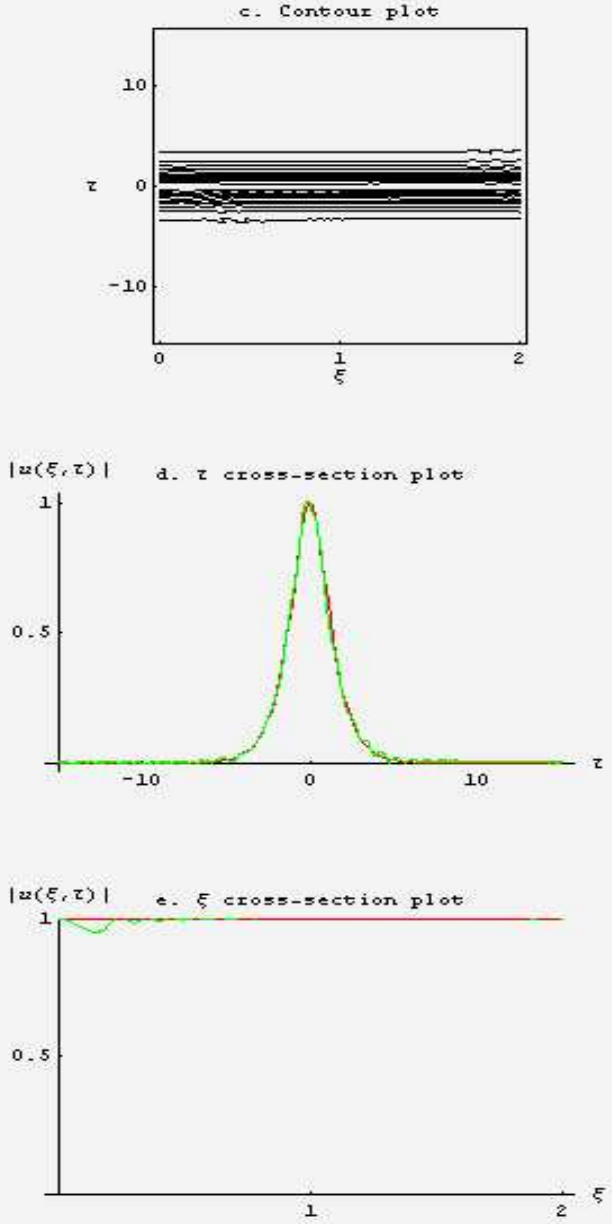


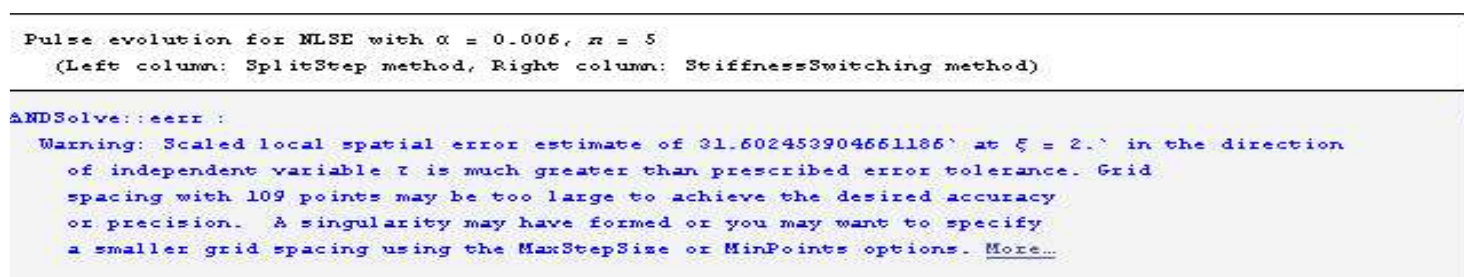

2. Forward amplitude plot

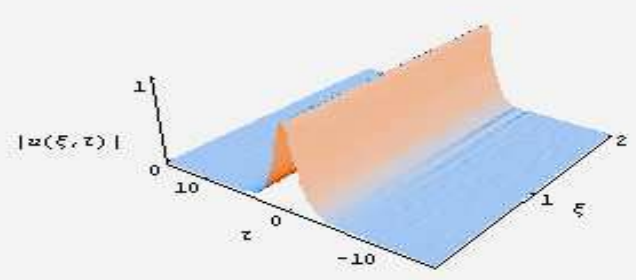

b. Reverse emplitude plot

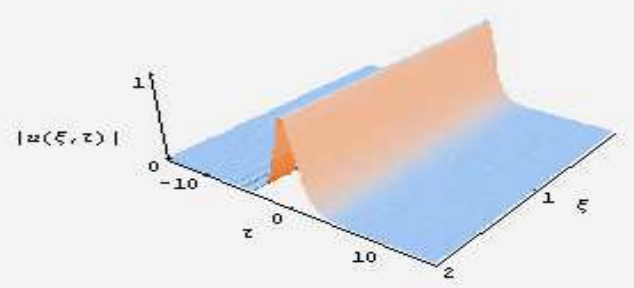

c. Contoux plot
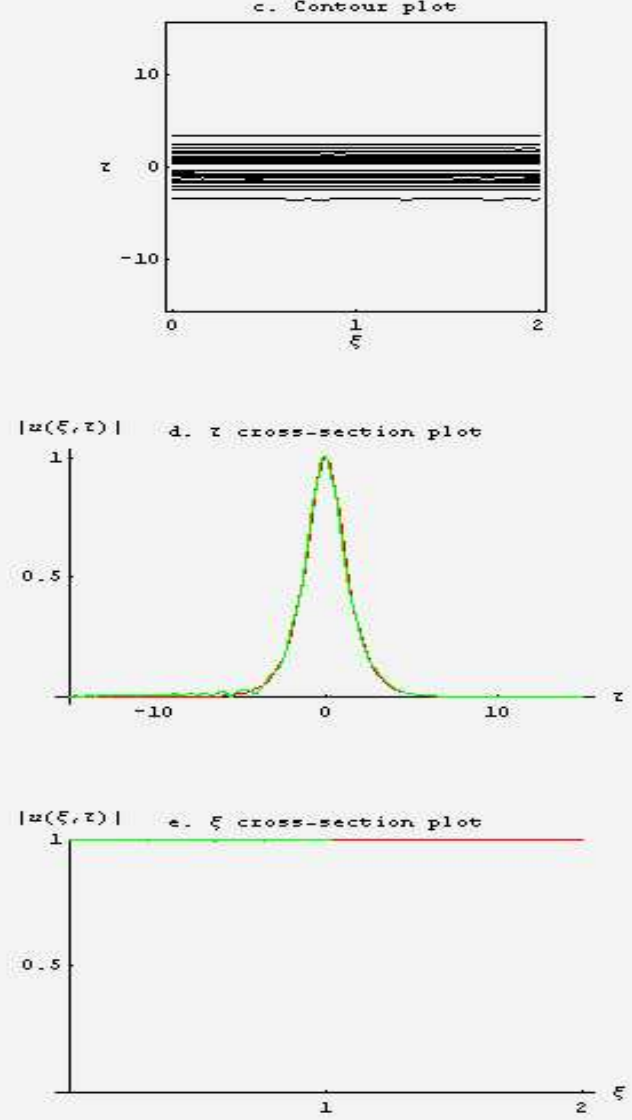

2. Forward amplitude plot

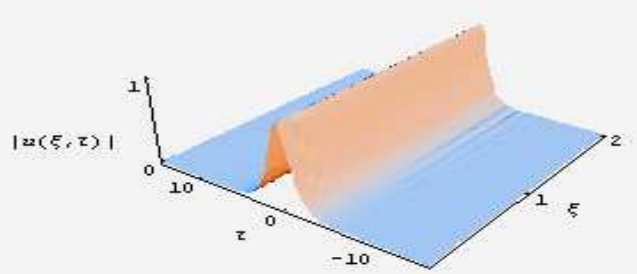

b. Reverse emplitude plot

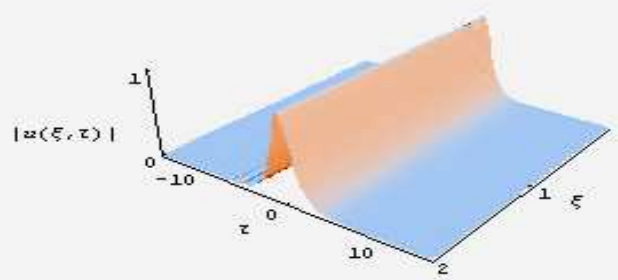

c. Contour plot

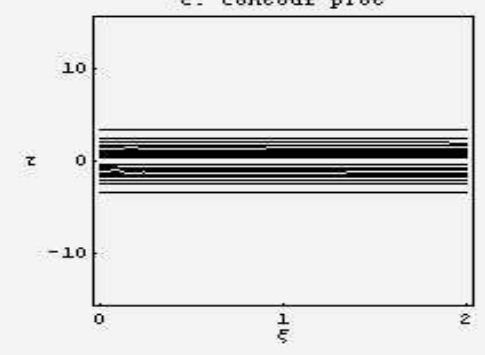

$|2(\xi, z)|$ d. $z$ exoss-section plot
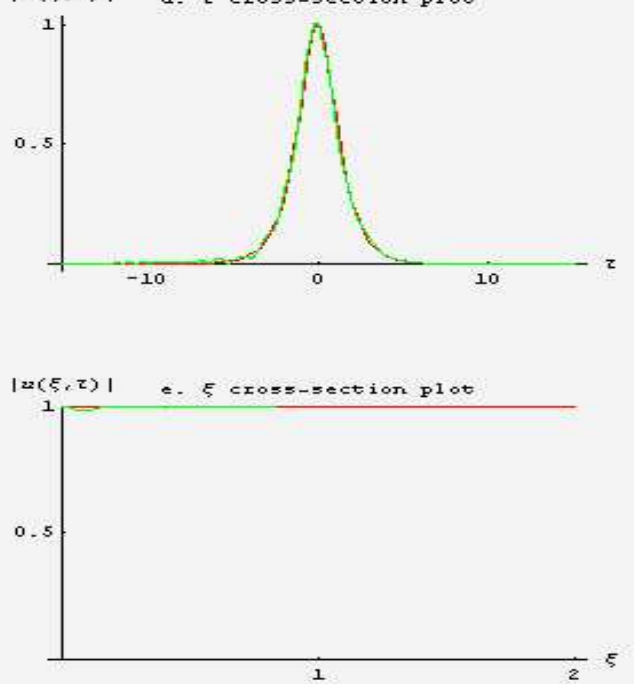
Pulse evolution for MLSE with $\alpha=0.0009, n=\delta$

(Left column: SplitStep method, Right column: Stiffresswitching method)

BGenera1: : ovfl: OVerflow occurred in computation. More..

AGerexal: :stop: Further output of General : ovfl will be suppressed duxing this ealculation. More...

2. Forward amplitude plot

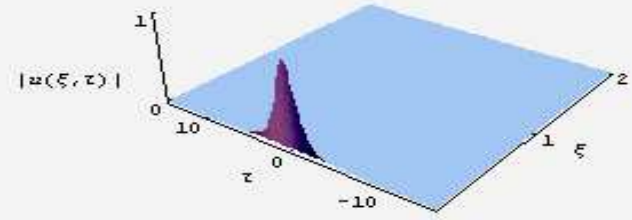

b. Reverse emplitude plot

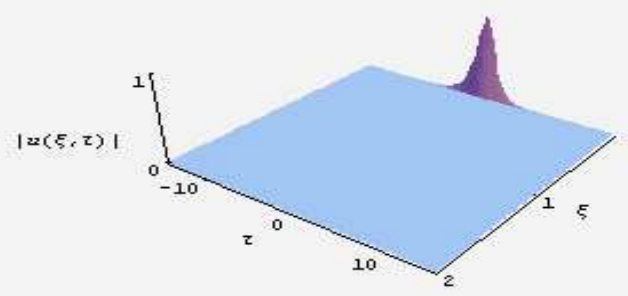

c. Contour plot

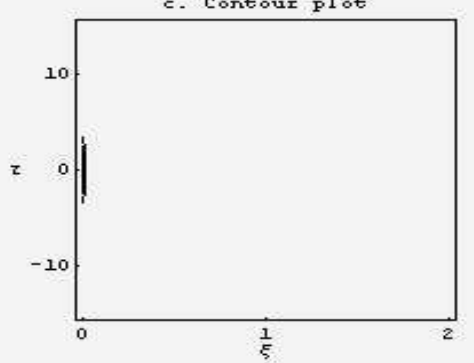

$|z(\xi, z)|$ d. $z$ exoss-section plot
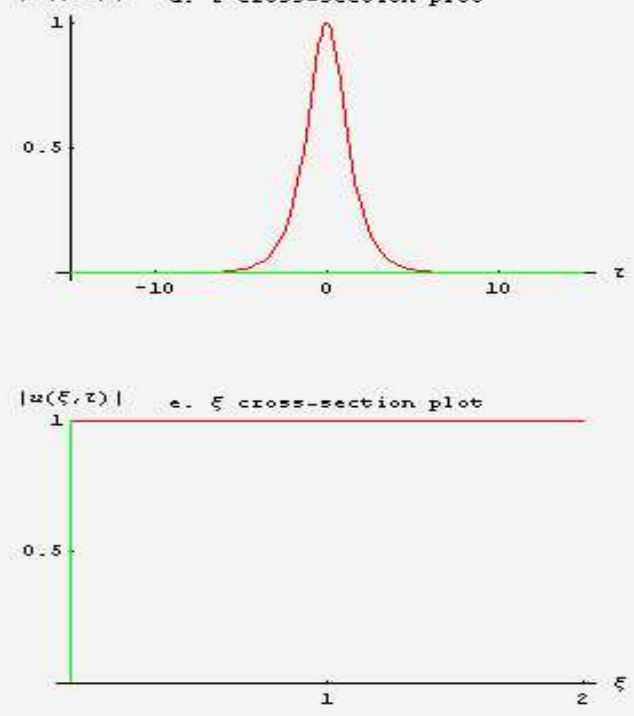

2. Forward amplitude plot

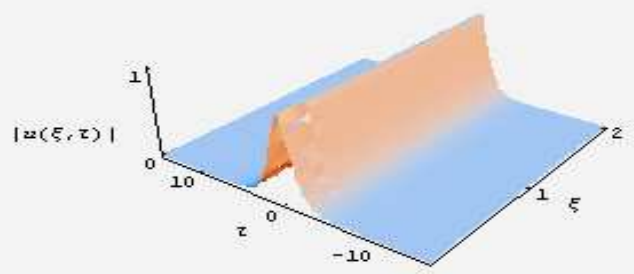

b. Reverses emplitude plot

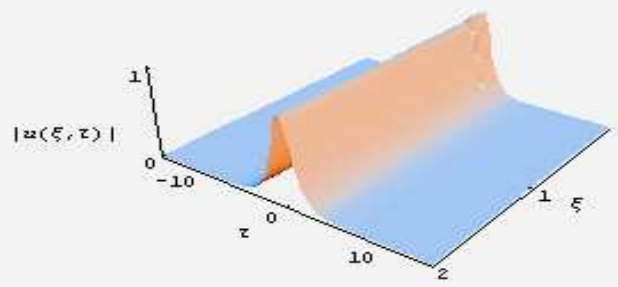

c. Contour plot
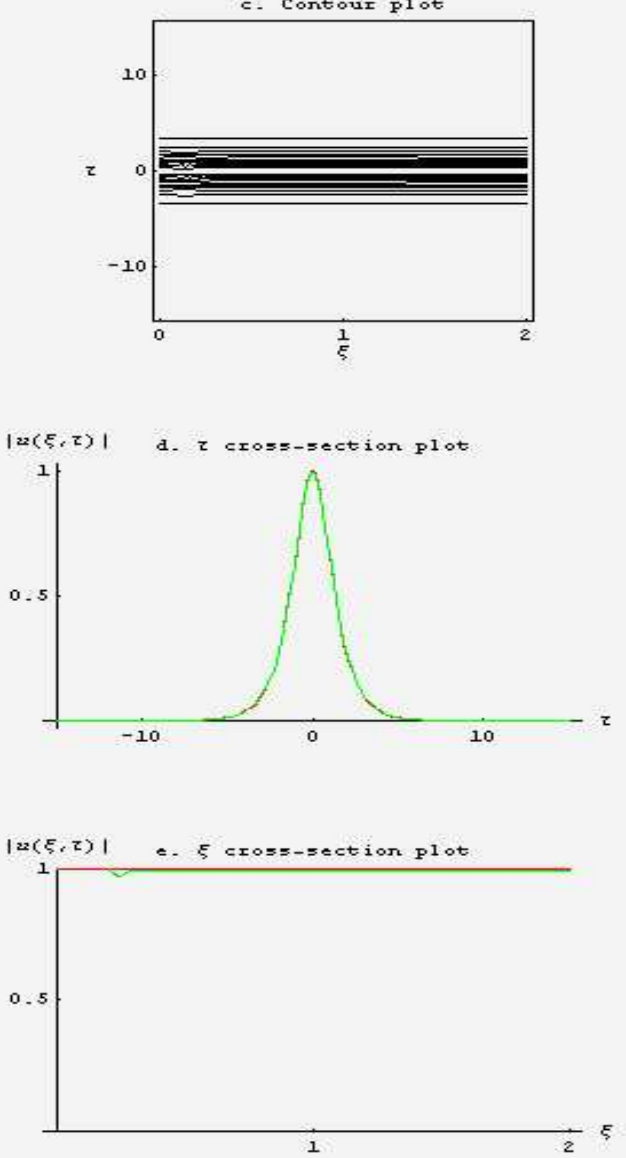


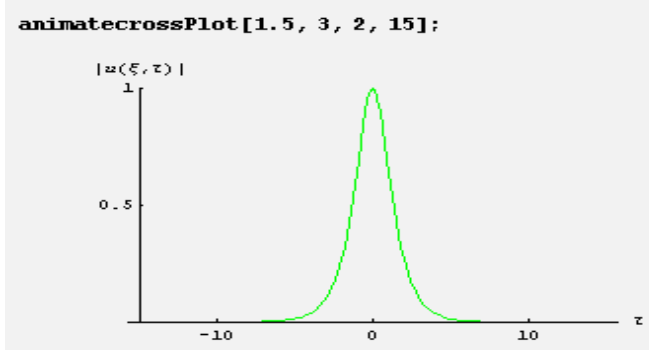

DISCUSSION

In the first set of plots; No dispersion, zero order, as was to be expected from the definition of a pure soliton wave, there was also no noticeable difference in the wave plots produced by the two methods under review here.

In the second case, part 1; Higher order dispersion, first order, coefficient (coef.) $\alpha=1$, we get another soliton solution. This time the wave is retarded, as exhibited by its angle to the $\tau$ axis in the amplitude and contour plots and its separation in the first crosssection. The second cross-section shows the extent of the retardation. In part 2 ; coef. $=1.8$, the previous observations are more marked, and here is observed an artifact in the StiffnessSwitching (St.Sw.) plots.

In the second order plots, coef. $=1.5$ the soliton solution still disperses symmetrically, and as predicted for an even order, shows less evidence of other activity, especially in SplitStep. However the accuracy of both methods now comes into question. Most other resemblance to a soliton has gone. The Split Step case has been severely retarded, as has the St.Sw., which is again showing the presence of numerical artefacts.

In the graphs representing the third order, the lower coef. $=0.05$ now shows the appearance of nonsymmetric radiations, in both methods, though minor at this coef., where retardation seems less evident. Moving up to the coef. $=1.5$ these effects become much more evident. Differences in the two methods are now evident, as is retarded. The radiation is seen to be feeding into the pulse and the question is now apparent as to whether this is due to nothing more than numerical artifacts.

Next is presented a short animation of the $\tau$ crosssection, which may be of some interest here. Coming now to the fourth order, the expectedly more peaceful background is again evident, though a small radiation, or artifact, may be seen. The resemblance to the classical definition of a soliton is, however markedly absent. In the Split Step the retardation is very severe and again raises serious doubts regarding the accuracy of this method.

For the fifth order non-convergence of the St.Sw. Method starts between coef. $=0.005$ and 0.006. Up to this level radiation is minor and retardation has not set in. The evident differences in the form of this "radiation" in the two methods again reinforce the argument for the belief that it is an artifact.
For the sixth order, any attempt at increasing the coef. Past 0.0009 initiates non-convergence. Up to this point however the same inconsistencies are clearly emerging, again bringing into question the reliability of either method.

\section{CONCLUSION}

There is no evidence here in the results produced using either the traditional StiffnessSwitching Method, or the newer Split Step method which would reinforce the premise that secondary radiation in the soliton wave exists in the quadratic range and not in the cubicle. The results produced here would even give ground to the assertion that secondary radiations do not occur at all!

The only conclusion that we can reach from this is that the methods provided in Mathematica to assist in the use of the NDSolve command are full of flaws, especially in respect to the presence, or the creation of numerical artifacts. From what we have observed with the Split Step method we can safely say that, while there are minor improvements, it does not instill sufficient confidence in the user that he might conclude the absence of artifacts here either.

\section{ACKNOWLEDGEMENT}

The authors acknowledge the assistance given to them in the production of this work by Dr Rob Knapp, Wolfram Research, for the use of his Mathematica package "Split Step. m. The authors also acknowledge Dr Paul Abbott, UWA for their invaluable input, which improved the results of this study. Associate Professor Richard Ollerton for his invaluable assistance and advice, with particular reference to the Mathematica coding used in this study.

\section{REFERENCES}

1. Hasegawa, A. and F. Tappert, 1973. Appl. Phys. Lett., 23: 142.

2. Herman, R.L., 1992. American Scientist, Jul.-Aug.

3. Doktorov, E.V., 2002. The modified nonlinear Schrodinger Equation: Facts and artifacts. Eur. Phys. J., 20: 227-231.

4. Zakharov and Shabat, 1972. Exact theory of twodimensional self-focusing and one-dimensional self-modulation of waves in nonlinear media. Soviet Physics JETP, 34: 62-69. 